\title{
Synergism in transcriptional activation: a kinetic view
}

\author{
Daniel Herschlag' and F. Brad Johnson \\ Department of Biochemistry, B400 Beckman Center, Stanford University, Stanford, California 94305-5307 USA
}

Synergistic action of transcriptional activators is common. Typically, these effects have been ascribed to equilibrium binding interactions. For example, two activators can act synergistically by binding to DNA cooperatively (e.g., Giniger and Ptashne 1988; Janson and Pettersson 1990; Xiao et al. 1991). An alternative equilibrium binding model for synergism entails an indirect interaction in which the two activators do not contact each other, but simultaneously bind to different sites within a single transcription complex (Carey et al. 1990; Lin et al. 1990). Although it seems likely that such direct and indirect physical interactions between transcriptional activators will account for many observed synergistic effects, it is possible that kinetic factors will provide synergism in some cases. Here, we present some possible models for "kinetic synergism," after introducing general functional and energetic descriptions of synergism.

Regulatory regions of eukaryotic genes often contain clusters of binding sites for several different activators (and repressors), with some of the activators (and repressors) having multiple binding sites (Dynan 1989). Synergism refers to a greater than additive increase in transcription when two or more activators are present simultaneously or when additional binding sites for a single activator are introduced (e.g., Martinez et al. 1987; Fromental et al. 1988; Han et al. 1989|. The observation of synergism demonstrates that the activators act in the same pathway, or in pathways that merge, to stimulate the production of transcript from a single DNA template molecule (Table 1). If they were acting independently to stimulate different transcription complexes, the largest possible amount of transcription from the two together would be the sum of the two pathways, with each activator increasing transcription as if the other were not present (Table 1). Thus, in the case of the interleukin-2 gene, the observation that NFAT and AP-1 are synergistic (Vacca et al. 1992) means that together they are facilitating a single transcriptional event. Synergism may play a general biological role in coordinating responses from many different signals and in allowing large responses from small changes in activator concentrations.

${ }^{1}$ Corresponding author.

\section{Energetic classes of synergism}

Synergistic effects can be grouped into three classes on the basis of the energetics of the specific synergistic event; these energetic classes are positive cooperativity, independent energetic effects, and negative cooperativity (Table 2). Thus, cooperativity is a subset of synergism. Examples of models for physical interactions that can give the three classes of energetic effects are shown schematically in Table 2. For positive cooperativity, activators A and B interact with each other as well as with the transcriptional apparatus, so that the presence of A strengthens the binding of $B$ and vice versa. [Positive cooperativity can also arise without direct interaction of $A$ and $B$; e.g., the first activator could orient the transcriptional complex to reduce the entropic cost for binding of the second activator, thereby enhancing binding of the second activator (not shown).] For energetic independence, activators $\mathrm{A}$ and $\mathrm{B}$ contact a common target, but do not interact with each other, nor does one affect the transcriptional apparatus to increase the affinity for the other: The binding site for $A$ is unaffected by the presence of $B$ and vice versa. Thus, the free energies for the interactions with $\mathrm{A}$ alone and with $\mathrm{B}$ alone are additive. This results in transcriptional levels with $A$ and $B$ present together that are the product of the levels with $\mathrm{A}$ alone and with $\mathrm{B}$ alone because rate and equilibrium constants are related logarithmically to free energy changes; mathematically, when energies are added, rates are multiplied (Table 2). For negative cooperativity, activators A and $B$ are shown as clashing (e.g., sterically or electrostatically|, so that the binding of one activator hinders binding of the other; nevertheless, these activators can behave synergistically. The above definitions of synergism and cooperativity provide a framework for the discussion of interactions in transcriptional activation but are different than some in the literature (e.g., Ptashne 1988; Carey et al. 1990); we reserve the term cooperativity to describe the energetics of particular classes of synergistic events rather than using these terms interchangeably.

It is important to point out that independence has a different meaning in Table 2 than in Table 1 ; this can be a source of confusion. The observation of synergism demonstrates that the activators act in the same pathway and are therefore not functionally independent $(\mathrm{Ta}$ - 
Table 1. Synergism occurs when two or more activators give transcriptional levels that are greater than the sum of the transcriptional levels from the individual activators (i.e., when transcription is greater than additive)

\begin{tabular}{|c|c|c|c|c|c|}
\hline & \multirow[b]{2}{*}{ Physical description } & \multirow[b]{2}{*}{ Simple model } & \multicolumn{3}{|c|}{ Level of transcription with } \\
\hline & & & A alone & B alone & $A$ and $B$ together \\
\hline Independent & separate pathways & & 10 & 10 & 20 \\
\hline Synergistic & $\begin{array}{l}A \text { and } B \text { act within } \\
\text { the same pathway }\end{array}$ & & 10 & 10 & $\begin{array}{c}100 \\
(\text { i.e., }>20)\end{array}$ \\
\hline
\end{tabular}

It should be noted that the absence of observed synergism does not prove that the activators act in separate pathways. For example, activators that act synergistically solely by (positive) cooperative binding (Table 2) would not display synergism if present at saturating concentrations because each activator would be fully bound in the absence of the other activator (see also Table 2, footnote b). For simplicity we consider the case in which there is no significant transcription in the absence of A or B. However, it should be noted that potential complications arise from basal levels. A related situation is described in footnote a of Table 2 .

ble 1). However, these functionally dependent activators can still be energetically independent; this energetic independence has significance for the type of interaction that is occurring within the pathway (Table 2). Independent pathways give additive amounts of transcription, whereas synergistic activators that are energetically independent give multiplicative amounts of transcription because the energetic contributions are additive (Tables 1 and 2).

\section{Kinetic synergism}

Physical models such as those in Table 2 provide the simplest conceptual explanations for synergism. However, there is a distinct class of mechanisms that can give rise to synergism through the enhancement of two or more slow or inefficient steps. We refer to this as kinetic synergism. Kinetic synergism can occur in the absence of direct or indirect physical interactions between the activators, and simultaneous physical association of the activators with the transcriptional complex is not required. [This possibility has been raised by Lin et al. (1990) and Li et al. (1991).] Nevertheless, this is perfectly in keeping with the above definition of synergism as denoting action within the same pathway. We note, however, that mechanistic distinctions for the different energetic classes of synergism are not readily drawn for kinetic synergism as they are for synergism originating from equilibrium binding effects (Table 2).

Below we consider three cases where transcriptional activators synergize without touching each other or simultaneously touching a common target. The examples are illustrative rather than exhaustive. For generality, we speak of "steps" that could in principle be any of those involved in transcription. Many steps are required for transcription by RNA polymerase II; each is a potential point of control by regulators. These steps are thought to include (1) the removal or modification of histones (for review, see Kornberg and Lorch 1991; Felsenfeld 1992); (2) the assembly of a preinitiation complex, which includes the polymerase and factors generally including TFIID (TBP and associated TAFs), TFIIA, TFIIB, TFIIE, TFIIF, TFIIH, and TFIIJ (for review, see Conaway and Conaway 1991; Zawel and Reinberg 1992); (3) two or more ATP-dependent events, including multiple phosphorylation of the carboxy-terminal domain of the bound polymerase (for review, see Peterson and Tjian 1992) and unwinding of the DNA to give an open complex (Wang et al. 1992a); and (4) initiation and elongation of the RNA transcript in the presence of NTPs. Thus, there is a plethora of steps that could be exploited to provide control and synergism using kinetic pathways similar to those described below.

\section{Case I}

Two steps in transcriptional initiation are each partially rate-limiting

In the transcriptional initiation reaction depicted in Figure $1 \mathrm{~A}$, there are two slow steps in the absence of activators. Addition of activator A greatly speeds step a (Fig. 1B), and addition of activator B greatly speeds step b (Fig. 1C). However, there is little change in the observed rate of initiation with either activator alone because a slow step remains; the reaction cannot proceed faster than the slowest step. It is therefore not until both activators are present and both slow steps are sped up that there is a large increase in the rate of initiation (Fig. 1D). The synergism of activators $A$ and $B$ does not require a physical connection; activator A could in principle dissociate before activator B exerts its effect. Thus, Figure 1 provides a scenario that can give kinetic synergism.

The scenario of Figure 1 can be generalized to situations where two or more molecules of the same activator are synergistic. However, this mechanism for kinetic 
Table 2. Energetic descriptions and hypothetical physical models of transcriptional synergism

\begin{tabular}{|c|c|c|c|c|c|c|c|}
\hline \multirow[b]{2}{*}{$\begin{array}{l}\text { Class and } \\
\text { physical model }\end{array}$} & \multirow[b]{2}{*}{$\begin{array}{l}\text { Energetic } \\
\text { description }\end{array}$} & \multicolumn{3}{|c|}{ Fold stimulation with } & \multicolumn{3}{|c|}{$-\Delta \Delta \mathrm{G}^{\text {relative to basal }}$} \\
\hline & & $\begin{array}{l}\text { A } \\
\text { alone }\end{array}$ & $\begin{array}{l}\text { B } \\
\text { alone }\end{array}$ & $\begin{array}{l}\mathrm{A} \text { and } \mathrm{B} \\
\text { together }\end{array}$ & $\begin{array}{l}\text { A } \\
\text { alone }\end{array}$ & $\begin{array}{l}\text { B } \\
\text { alone }\end{array}$ & $\begin{array}{l}A \text { and } B \\
\text { together }\end{array}$ \\
\hline Positive cooperativity & $\begin{array}{l}\text { greater than additive } \\
\text { in free energy }\end{array}$ & 10 & 10 & $>100$ & 1.3 & 1.3 & $>2.6$ \\
\hline Independent energetic effects & additive in free energy & 10 & 10 & 100 & 1.3 & 1.3 & 2.6 \\
\hline cooperativity & $\begin{array}{l}\text { less than additive } \\
\text { in free energy }\end{array}$ & 10 & 10 & $21-99^{b}$ & 1.3 & 1.3 & $1.8-2.5^{\mathrm{c}}$ \\
\hline
\end{tabular}

The schematic physical models represent examples that give each class of energetic effects and are described in the text.

${ }^{a}$ The values of $-\Delta \Delta \mathrm{G}^{\text {relative to basal }}$ are in units of $\mathrm{kcal} / \mathrm{mole}$ and were calculated from the relationship $\Delta \Delta \mathrm{G}=-\mathrm{RT} \operatorname{lnk} \mathrm{rel}_{\mathrm{rel}} l=(1.3$ $\mathrm{kcal} / \mathrm{mole}$ ) $\times \log k_{\mathrm{rel}}$ at $25^{\circ} \mathrm{C}$ ) (Laidler 1978). Thus, every 10 -fold increase in the rate of transcription corresponds to $1.3 \mathrm{kcal} / \mathrm{mole}$ of favorable or stabilizing free energy (i.e., $\Delta \Delta \mathrm{G}$ is negative). For example, the 100 -fold stimulation from "Independent energetic effects" gives $2 \times 1.3 \mathrm{kcal} / \mathrm{mole}=2.6 \mathrm{kcal} / \mathrm{mole}$ of stabilization because transcription is stimulated by two factors of 10 (i.e., $10^{2}$-fold). The

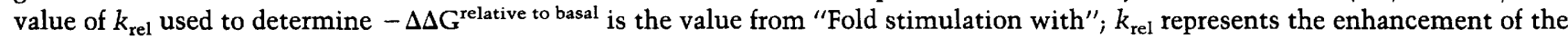
transcription rate above the basal rate that is obtained in the absence of the activators. A technical, but important point is that background or basal effects can "mask" or obscure the simple physical interpretations of the energetic effects presented. For example, consider experimental background or transcription from a different pathway, one that is not responsive to the activators. This pathway might use a subset of the transcription factors that are used in the activator-responsive pathway. This would give an observed basal level of transcription that is higher than that which would be obtained in the absence of the second pathway. The fold stimulation for the pathway of interest would then be masked, with the fold stimulation observed experimentally on addition of activator A or B alone less than the fold stimulation of the pathway of interest. Thus, the fold stimulation when A and B are present together will be artificially large relative to the masked stimulation with A or B alone. Unfortunately, this can result in qualitatively different conclusions, e.g., observing apparent positive cooperativity that originates from negative cooperativity on the molecular level.

'There are also physical models in which negative cooperativity would give transcriptional levels of $<20$, i.e., less than numerically additive so that a synergistic enhancement of transcription would not be seen, even though enhancers $A$ and $B$ were interacting within the same transcriptional complex. For example, if activators A and B are both present at saturating concentrations so that both are always bound, the presence of activator A might interfere with activation by $B$ and vice versa, thereby giving a smaller enhancement of transcription than the sum of transcription with $\mathrm{A}$ alone and with $\mathrm{B}$ alone.

${ }^{\mathrm{c}}$ The value of $1.8 \mathrm{kcal} / \mathrm{mole}$ as the lower limit for a synergistic effect comes from the fact that the numerically additive case that gives 20 -fold stimulation corresponds to $1.7 \mathrm{kcal} / \mathrm{mole}$. [This comes from $-\Delta \Delta \mathrm{G}=(1.3 \mathrm{kcal} / \mathrm{mole}) \times \log k_{\text {rel }}=(1.3 \mathrm{kcal} / \mathrm{mole} \times \log 20=$ $1.7 \mathrm{kcal} / \mathrm{mole}$ (see a).]

synergism with two copies of the same activator is operative only if the two activators exert different effects on the two steps. This can arise because the activators affect different steps from their different locations or because, after affecting step a (Fig. 1), the first activator is sequestered and cannot act in step $b$. If instead both activators have the same effect on each step, then kinetic synergism does not arise from this mechanism. Green and coworkers have suggested that one molecule of the activator VP-16 could enhance the stability of TFIID binding (Stringer et al. 1990; Ingles et al. 1991) and another molecule of VP-16 could enhance the stability of TFIIB binding (Lin and Green 1991; Lin et al. 1991). Analogously, steps $a$ and $b$ in Figure 1 could represent TFIID- and TFIIB-dependent steps, respectively, that are enhanced by two VP-16 molecules to give kinetic synergism.
Finally, the facilitation of the separate steps need not be from positive effects, but could instead result from anti-inhibitory effects. For example, two repressors might need to be displaced to allow transcription to proceed; in this model, each repressor alone is sufficient to effectively shut down transcription. It is not until both activators are present that transcription can proceed at a reasonable rate. Thus, the activators behave synergistically, again without a requirement for a physical connection.

\section{Case II}

Branches in the pathway for transcriptional initiation

When transcriptional complexes are assembled under basal conditions in vitro, only a small fraction of the 
Figure 1. Kinetic synergism with two steps in transcriptional initiation partially rate-limiting. (A) Basal transcription. (B) Transcription in the presence of activator $A$, which speeds step a by 100 -fold but has only a $\sim 2$ fold effect on the overall rate of initiation. $|C|$ Transcription in the presence of activator $B$, which speeds step b by 100 -fold but also has only a $\sim 2$-fold effect on the overall rate of initiation. (D) Transcription in the presence of both activators results in a large increase in the rate of initiation, larger than the sum of the initiation with each activator alone. Thus, activators A and B act synergistically. The rate constants were calculated from the equation

$$
1 / k_{\text {obsd }}=\Sigma\left(1 / k_{\mathrm{i}}\right)
$$

where $k_{\mathrm{i}}$ represents the rate constants for the individual steps. For simplicity, steps $\mathrm{a}$ and $\mathrm{b}$ are shown as irreversible. For reversible reactions, the method of "net rate constants" can be utilized to derive an analogous expression so that kinetic synergism is still obtained (Cleland 1974; Fersht 1985).
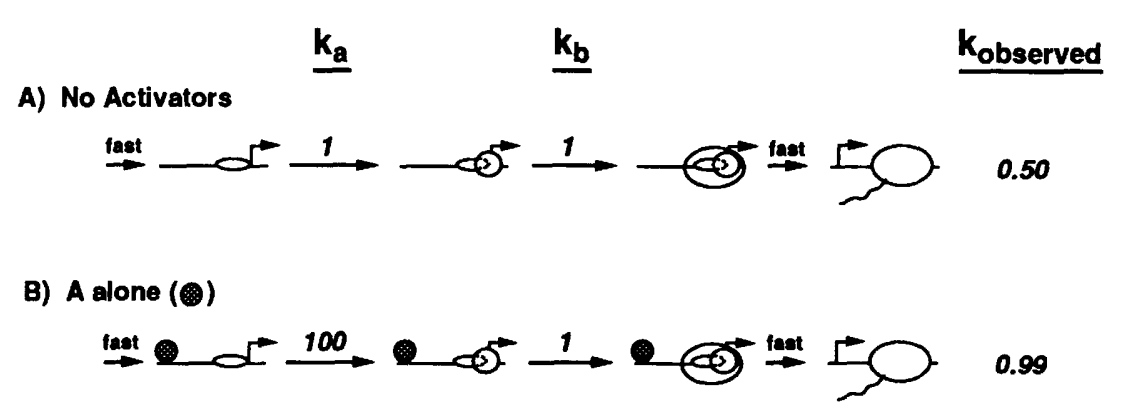

C) B alone ( $\mathbf{T})$

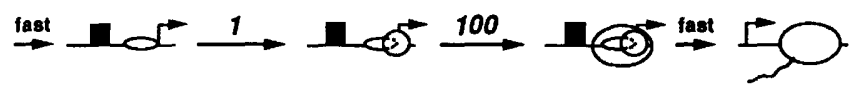

0.99

D) A \& B together

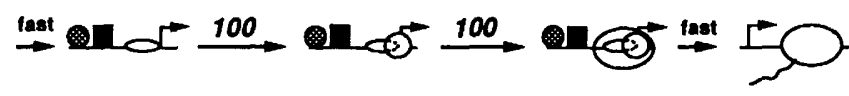

50
DNA templates are active in each round of transcription (Hawley and Roeder 1987; Kadonaga 1990; for a possible exception, see Van Dyke et al. 1988), even though footprinting experiments have shown that most templates are bound within complexes (Horikoshi, et al. 1988; Van Dyke et al. 1988; Maldonado et al. 1990). These results are consistent with branches or decision points at which the templates partition between the formation of productive complexes and the formation of nonproductive complexes. Furthermore, transcriptional activators /and repressors) have been shown to exert an effect on the final extent of productive preinitiation complex formation, rather than on the observed rate constant for formation of productive complexes (Carcamo et al. 1989; Schmidt et al. 1989; Ayer and Dynan 1990; Katagiri et al. 1990; Johnson and Krasnow 1992; White et al. 1992). Addition of activators or repressors (Workman et al. 1990; Zhu et al. 1991; Johnson and Krasnow 1992; Wang et al. 1992b) or dilution (Hawley and Roeder 1985) after assembly of the preinitiation complex does not affect this extent, implicating a branched pathway rather than an equilibrium effect. The transcriptional inhibitors NC2 and Drl may be acting at such branchpoints, sequestering the complex in an inactive state (Meisterernst and Roeder 1991; Inostroza et al. 1992). Although it is not known whether there are branches during transcriptional initiation in vivo, these in vitro results are provocative.

Activators that act at different branchpoints can give kinetic synergism (Fig. 2). Each of the two branchpoints in Figure 2 allows a choice between continuing along the initiation pathway or branching to form a nonproductive complex. The extent of productive complex formation is then determined by the relative rates for partitioning be- tween the productive and nonproductive paths $\left(k_{\mathrm{A}}\right.$ vs. $k_{\mathrm{i}}$ and $k_{\mathrm{B}}$ vs. $\left.k_{\mathrm{i}}{ }^{\prime}\right)$. Activator $\mathrm{A}$ or $\mathrm{B}$ alone has only a small effect on the formation of productive initiation complex. This is because even if activator A allows nearly all of the complex to proceed toward productive complex in the first step, without activator B most of this potentially productive complex is lost in the second partitioning step. It is not until activators A and B are both present that there is a large increase in the amount of the productive complex that is formed. Thus, activators $\mathrm{A}$ and $B$ act synergistically while exerting their effects on distinct steps, without a requirement for a direct or indirect physical connection. Synergism can also result with branched pathways when two identical activators facilitate both steps in Figure 2, unlike case I. The effect is amplified by repeated use of the activator; this is analogous to the effect described in case III below. The amount of synergism with identical activators can then be enhanced if there are more than two branched steps. The analysis of Figure 2 emphasizes another basic point: Kinetic partitioning at branches can control initiation even when the steps at the branches are not the slowest steps in initiation (i.e., not the rate-limiting steps). Thus, it is important to seperate effects on the rate of initiation from effects on the extent of initiation. Furthermore, the observation of branched pathways may require reevaluation of data that were interpreted solely in terms of rate-limiting steps and equilibrium processes.

Branched pathways can also provide proofreading, a mechanism that provides greater control that is paid for through an expenditure of energy (Hopfield 1974; Fersht 1985; Yarus 1992). Figure 2 presents one of several possibilities for proofreading. Here, the steps in formation of the productive and nonproductive preinitiation com- 


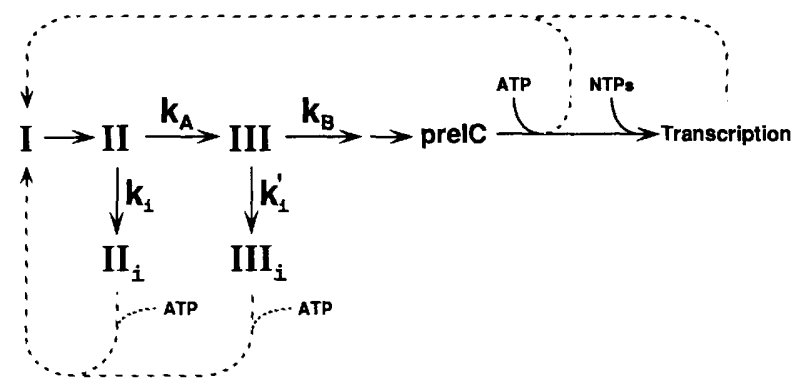

\begin{tabular}{|c|c|c|c|}
\hline Activator & $\mathbf{k}_{\mathbf{A}}$ & $\mathbf{k}_{\mathrm{B}}$ & Transcriptional Level \\
\hline A & 10 & 0.1 & 0.08 \\
\hline B & 0.1 & 10 & 0.08 \\
\hline$A \& B$ & 10 & 10 & 0.83 \\
\hline
\end{tabular}

Figure 2. Kinetic synergism with two branchpoints in the pathway for transcriptional initiation. At each branch there is a choice between continuing along the pathway of transcriptional initiation or forming a nonproductive complex. The rate constants $k_{\mathrm{A}}$ and $k_{\mathrm{B}}$ are independently stimulated by activators $\mathrm{A}$ and $B$, respectively. The level of transcription in the presence of activator $\mathrm{A}$ alone, $\mathrm{B}$ alone, and $\mathrm{A}$ and $\mathrm{B}$ together is summarized. These levels were calculated with the rate constants for inactivation $k_{\mathrm{i}}$ and $k_{\mathrm{i}}{ }^{\prime}$ both equal to 1 and the rate constants $k_{\mathrm{A}}$ and $k_{\mathrm{B}}$ as listed. The transcriptional level is the fraction of complex that proceeds successfully through both branchpoints [i.e., transcriptional level $=\left(k_{\mathrm{A}} /\left(k_{\mathrm{A}}+k_{\mathrm{i}}\right) \times\left(k_{\mathrm{B}} /\left(k_{\mathrm{B}}+k_{i}{ }^{\prime}\right)\right)\right.$. For simplicity, the transcriptional level in the absence of activators is negligible and the rate constant for recycling to the beginning of the assembly process is taken to be the same for the productive and nonproductive complexes. The productive and nonproductive complexes require ATP to recycle, so that the decision (for that initiation event) made at the branch is irreversible. This can allow proofreading, as described in the text.

plexes are essentially irreversible due to, for example, a large favorable binding free energy in the direction of complex formation. Subsequently, energy from the hydrolysis of ATP is used to disassemble and recycle the nonproductive complexes; the productive complexes are recycled both in the proofreading step and following initiation. There is evidence for an ATP requirement in the recycling of productive and nonproductive complexes assembled in vitro (Cai and Luse 1987; Johnson and Krasnow 1992).

It is possible to imagine several potential roles for transcriptional proofreading. However, it is important to emphasize that the following ideas are speculative as we are aware of no data supporting transcriptional proofreading in vivo.

1. Proofreading could provide a mechanism to correct "mistakes" that lead to the formation of misassembled and thus nonproductive transcriptional complexes.

2. Proofreading could provide greater control, especially by preventing genes being turned on when their gene products are deleterious. This would be analogous to proofreading in replication and translation, which limit base misincorporation into DNA and amino acid misincorporation into proteins (Kunkel 1988; Thompson 1988).

3. Multiple branches within a proofreading pathway (Fig. 2) could allow different regulatory factors to influence different decision points for a single transcriptional event. Convergence of the regulatory factors through action at distinct decision points could obviate a requirement to have several distinct regulatory pathways affect a common signal molecule which, in turn, regulates transcription. Such mechanisms might be especially useful in a case where a gene product is undesirable unless several regulatory criteria are met simultaneously.

\section{Case III}

\section{Stimulation of a protein kinase by transcriptional activators}

Phosphorylation of the carboxy-terminal domain (CTD) of RNA polymerase II has been correlated with the initiation of transcription (Laybourn and Dahmus 1990; Arias et al. 1991), and a protein from a highly purified fraction is required for both transcription and CTD kinase activity (Gileadi et al. 1992; Lu et al. 1992). There is also evidence for phosphorylation of many transcriptional regulators such as c-Jun and CREB (Hunter and Karin 1992). Thus, it seems reasonable to consider possible roles for phosphorylation in the control of transcriptional initiation.

Multiple phosphorylation events during initiation can result in kinetic synergism. One scenario for this is presented in Figure 3, where three phosphorylations are required for initiation and each phosphorylation event is opposed by the action of a phosphatase. Activators A and $B$ each stimulate a kinase. This stimulation gives a larger concentration of the complex with three covalently bound phosphates, thereby increasing transcription. With activators $A$ and $B$ present together, the kinase activity (i.e., $k_{1}, k_{2}$, and $k_{3}$ in Fig. 3 ) is simply the sum of that with $A$ and $B$ alone. This is because the activators do not interact with one another, but are functionally independent in stimulating phosphorylation. (This could arise from stimulation of the same or different kinases.) However, the additive effect on the individual rate constants is amplified in the overall transcription reaction because it is repeated in several steps. The resultant greater-than-additive increase in transcription thus arises via kinetic synergism.

Two (or more) copies of the same activator also give synergism according to the model in Figure 3, as would a model with only two phosphorylation events (though the synergism would be less pronounced). In addition, the partially rate-limiting steps in case I and the branchpoints in case II could represent phosphorylation events, so that phosphorylation could be involved in a variety of kinetic synergism models. Finally, it should be noted 


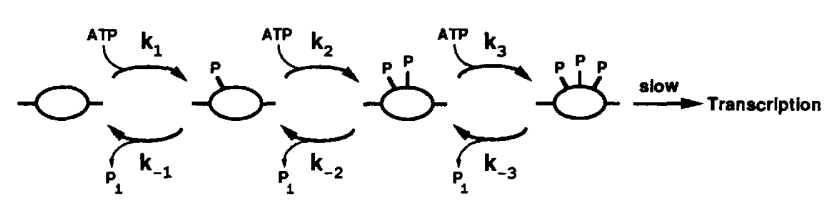

$\begin{array}{cccccc}\text { Activator } & & \frac{k_{1}}{1} & \frac{k_{2}}{k_{2}} & \frac{k_{3}}{1} & \frac{\text { Transcriptional level }}{9 \times 10^{-4}} \\ \text { B } & 1 & 1 & 1 & 9 \times 10^{-4} \\ \text { A \& B } & 1 & 1 & 1 & 64 \times 10^{-4}\end{array}$

Figure 3. Kinetic synergism with three phosphorylation events required for transcription. Each phosphorylation $\left\{k_{1}, k_{2}\right.$, $\left.k_{3}\right)$ is opposed by the action of a phosphatase $\left(k_{-1}, k_{-2}, k_{-3}\right)$. The level of transcription in the presence of activator A alone, $\mathrm{B}$ alone, and $\mathrm{A}$ and $\mathrm{B}$ together is summarized. These levels were calculated with the rate constants for phosphorylation listed and the rate constants for dephosphorylation, $k_{-1}, k_{-2}$ and $k_{-3}$, all equal to 10. A step after phosphorylation is slow in this model so that the level of transcription is directly proportional to the steady-state concentration of the complex with three covalently linked phosphates. (This concentration is calculated from the steady-state expressions $\mathrm{d}[X] / \mathrm{dt}=0=-k_{1}[X]$ $+k_{-1}[X-P] ; \mathrm{d}[X-P] / \mathrm{dt}=0=k_{1}[X]+k_{-2}\left[X-P_{2}\right]-\left(k_{-1}+\right.$ $\left.k_{2}\right)[X-P]$; etc., and the conservation equation fraction $(X)+$ fraction $(X-P)+$ fraction $\left(X-P_{2}\right)+$ fraction $\left(X-P_{3}\right)=1$. For simplicity, the level of transcription in the absence of either activator is considered negligible (i.e., $k_{1}, k_{2}$, and $k_{3}$ very small). Also for simplicity, the pathway of phosphorylation is shown as ordered.

that there are many types of indirect effects, analogous to stimulation of a kinase that then acts in transcriptional initiation. These other indirect effects can also result in kinetic synergism when, as in Figure 3, multiple steps are affected simultaneously.

\section{Perspective}

Synergism between transcriptional activators is frequently modeled in terms of direct or indirect physical interactions. In principle, however, there could also be kinetic synergism, in which the activators are physically distincty in their action but nevertheless give greaterthan-additive stimulation when present together. Given the large number of factors and steps involved in transcription, it would seem remarkable to us if there were not some cases of kinetic synergism. Kinetic synergism may provide a solution to the problem of having to accommodate many regulators acting on a single gene, as is common in eukaryotes. Not only can the regulators simultaneously touch different parts of the transcriptional complex, but different regulators might be used at different times in the transcriptional initiation pathway. In addition, the issues discussed herein may be generally applicable to other multistep processes, such as spliceosomal assembly in pre-mRNA splicing (Guthrie 1991).

Distinguishing the broad classes of physical and ki- netic synergism will require dissection and analysis of the individual steps in transcriptional activation and initiation. In turn, investigations of synergism may be useful probes of fundamental aspects of transcription.

\section{Acknowledgments}

We thank Tom Cech and Mark Krasnow for many stimulating discussions, for comments on the manuscript, and for their strong encouragement. We also thank Bob Kingston, Mike Botchan, and Steve Hahn for helpful comments on the manuscript. This work was supported by a grant from the Lucille P. Markey Charitable Trust to D.H. D.H. is a Lucille P. Markey Scholar in Biomedical Science. F.B.J. is a Medical Scientist Trainee of the National Institute of General Medical Sciences (2T32GM07365).

\section{References}

Arias, J.A., S.R. Peterson, and W.S. Dynan. 1991. Promoter-dependent phosphorylation of RNA polymerase II by a template-bound kinase. J. Biol. Chem. 266: 8055-8061.

Ayer, D.E. and W.S. Dynan. 1990. A downstream-element-binding factor facilitates assembly of a functional preinitiation complex at the simian virus 40 major late promoter. Mol. Cell. Biol. 10: 3635-3645.

Cai, H. and D.S. Luse. 1987. Transcription initiation by RNA polymerase II in vitro. I. Biol. Chem. 262: 298-304.

Carcamo, J., S. Lobos, A. Merino, L. Buckbinder, R. Weinmann, V. Nataragan, and D. Reinberg. 1989. Factors involved in specific transcription by mammalian RNA polymerase II. Role of factors IID and MLTF in transciption from the Adenovirus major late and IVa2 promoters. I. Biol. Chem. 264: 7704-7714.

Carey, M., Y.S. Lin, M.R. Green, and M. Ptashne. 1990. A mechanism for synergistic activation of a mammalian gene by GAL4 derivatives. Nature 345: 361-364.

Cleland, W.W. 1974. Partition analysis and the concept of net rate constants as tools in enzyme kinetics. Biochemistry 14: $3220-3224$.

Conaway, J.W. and R.C. Conaway. 1991. Initiation of eukaryotic messenger RNA synthesis. I. Biol. Chem. 266: 1772117724.

Dynan, W.S. 1989. Modularity in promoters and enhancers. Cell 58: $1-4$.

Felsenfeld, G. 1992. Chromatin as an essential part of the transcriptional mechanism. Nature 355: 219-224.

Fersht, A.M. 1985. Enzyme structure and mechanism, 2nd ed., pp. 117-120. Freeman, New York.

Fromental, C., M. Kanno, H. Nomiyama, and P. Chambon. 1988. Cooperativity and hierarchical levels of functional organization in the SV40 enhancer. Cell 54: 943-953.

Gileadi, O., W.J. Feaver, and R.D. Kornberg. 1992. Cloning of a subunit of yeast RNA polymerase II transcription factor $b$ and CTD kinase. Science 257: 1389-1392.

Giniger, E. and M. Ptashne. 1988. Cooperative DNA binding of the yeast transcriptional activator GAL4. Proc. Natl. Acad. Sci. 85: 382-386.

Guthrie, C. 1991. Messenger RNA splicing in yeast: Clues to why the spliceosome is a ribonucleoprotein. Science 253: 153-163.

Han, K., M.S. Levine, and J.L. Manley. 1989. Synergistic activation and repression of transcription by Drosophila homeobox proteins. Cell 56: 573-583.

Hawley, D.K. and R.G. Roeder. 1985. Separation and partial 
characterization of three functional steps in transcription initiation by human RNA polymerase II. J. Biol. Chem. 260: 8163-8172.

-1987. Functional steps in transcription initiation and reinitiation from the major late promoter in a HeLa nuclear extract. J. Biol. Chem. 262: 3452-3461.

Hopfield, J.J. 1974. Kinetic proofreading: A new mechanism for reducing errors in biosynthetic processes requiring high specificity. Proc. Natl. Acad. Sci. 71: 4135-4139.

Horikoshi, M., T. Hai, Y.-S. Lin, M.R. Green, and R.G. Roeder. 1988. Transcription factor ATF interacts with the TATA factor to facilitate establishment of a preinitiation complex. Cell 54: 1033-1042.

Hunter, T. and M. Karin. 1992. The regulation of transcription by phosphorylation. Cell 70: 375-387.

Ingles, C.J., M. Shales, W.D. Cress, S.J. Triezenberg, and J. Greenblatt. 1991. Reduced binding of TFIID to transcriptionally compromised mutants of VP16. Nature 351: 588-590.

Inostroza, J.A., F.H. Mermelstein, I. Ha, W.S. Lane, and D. Reinberg. 1992. Drl, a TATA-binding protein-associated phosphoprotein and inhibitor of class II gene transcription. Cell 70: 477-489.

Janson, L. and U. Pettersson. 1990. Cooperative interactions between transcription factors Spl and OTF-1. Proc. Natl. Acad. Sci. 87: 4732-4736.

Johnson, F.B. and M.A. Krasnow. 1992. Differential regulation of transcription preinitiation complex assembly by activator and repressor homeodomain proteins. Genes \& Dev. 6: $2177-2189$.

Kadonaga, J.T. 1990. Assembly and disassembly of the Drosophila RNA Polymerase II complex during transcription. $J$. Biol. Chem. 265: 2624-2631.

Katagiri, F., K. Yamazaki, M. Horikoshi, R.G. Roeder, and N.-H. Chua. 1990. A plant DNA-binding protein increases the number of active preinitiation complexes in a human in vitro transcription system. Genes \& Dev. 4: 1899-1909.

Kornberg, R.D. and Y. Lorch. 1991. Irresistible force meets immovable object: Transcription and the nucleosome. Cell 67: 833-836.

Kunkel, T.A. 1988. Exonucleolytic proofreading. Cell 53: 837840.

Laidler, K.J. 1978. Physical Chemistry with Biological Applications, pp. 391-398. Benjamin/Cummings Menlo Park, CA.

Laybourn, P.J. and M.E. Dahmus. 1990. Phosphorylation of RNA polymerase IIA occurs subsequent to interaction with the promoter and before the initiation of transcription. I. Biol. Chem. 265: 13165-13173.

Li, R., J.D. Knight, S.P. Jackson, R. Tjian, and M.R. Botchan. 1991. Direct interaction between Spl and the EPV enhancer E2 protein mediates synergistic activation of transcription. Cell 65: 493-505.

Lin, Y.-S. and M.R. Green. 1991. Mechanism of action of an acidic transcriptional activator in vitro. Cell 64: 971-981.

Lin, Y.-S., M. Carey, M. Ptashne, and M.R. Green. 1990. How different eukaryotic transcriptional activators can cooperate promiscuously. Nature 345: 359-361.

Lin, Y.-S., I. Ha, E. Maldonado, D. Reinberg, and M.R. Green. 1991. Binding of general transcription factor TFIIB to an acidic activating region. Nature 353: $569-571$.

Lu, H., L. Zawel, L. Fisher, J.-M. Egly, and D. Reinberg. 1992. Human general transcription factor IIH phosphorylates the carboxy-terminal domain of RNA polymerase II. Nature 358: $641-645$.

Maldonado, E., I. Ha, P. Cortes, L. Weis, and D. Reinberg. 1990. Factors involved in specific transcription by mammalian RNA polymerase II: Role of transcription factors IIA, IID, and IIB during formation of a transcription-competent complex. Mol. Cell. Biol. 10: 6335-6347.

Martinez, E., F. Givel, and W. Wahli. 1987. The estrogen-responsive element as an inducible enhancer: DNA sequence requirements and conversion to a glucocorticoid-responsive element. EMBO /. 6: 3719-3727.

Meisterernst, M. and R.G. Roeder. 1991. Family of proteins that interact with TFIID and regulate promoter activity. Cell 67: 557-567.

Peterson, G.M. and R. Tjian. 1992. The tell-tail trigger. Nature 358: 620-621.

Ptashne, M. 1988. How eukaryotic transcriptional activators work. Nature 335: 683-689.

Schmidt, M.C., Q. Zhou, and A.J. Berk. 1989. Spl activates transcription without enhancing DNA-binding activity of the TATA box factor. Mol. Cell. Biol. 9: 3299-3307.

Stringer, K.F., C.J. Ingles, and J. Greenblatt. 1990. Direct and selective binding of an acidic transcriptional activation domain to the TATA-box factor TFIID. Nature 345: 783-786.

Thompson, R.C. 1988. EFTu provides an internal kinetic standard for translational accuracy. Trends Biochem. Sci. 13: 9193.

Vacca, A., M.P. Felli, A.R. Farina, S. Martinotti, M. Maroder, I. Screpanti, D. Meco, E. Petrangeli, L. Frati, and A. Gulino. 1992. Glucocorticoid receptor-mediated suppression of the interleukin 2 gene expression through impairment of the cooperativity between nuclear factor of activated $\mathrm{T}$ cells and AP-1 enhancer elements. I. Exp. Med. 175: 637-646.

Van Dyke, M.W., R.G. Roeder, and M. Sawadogo. 1988. Physical analysis of transcription preinitiation complex assembly on a class II gene promoter. Science 241: 1335-1338.

Wang, W., M. Carey, and J.D. Gralla. 1992a. Polymerase II promoter activation: Closed complex formation and ATPdriven start site opening. Science 255: 450-453.

Wang, W., J.D. Gralla, and M. Carey. 1992b. The acidic activator GAL4-AH can stimulate polymerase II transcription by promoting assembly of a closed complex requiring TFIID and TFIIA. Genes \& Dev. 6: 1716-1727.

White, J., C. Brou, J. Wu, Y. Lutz, V. Moncollin, and P. Chambon. 1992. The acidic transcriptional activator GAL-VP16 acts on preformed template-committed complexes. EMBO J. 11: $2229-2240$.

Workman, J.L., R.G. Roeder, and R.E. Kingston. 1990. An upstream transcription factor, USF (MLTF) facilitates the formation of preinitiation complexes during in vitro chromatin assembly. EMBO I. 9: 1299-1308.

Xiao, H., O. Perisic, and J.T. Lis. 1991. Cooperative binding of Drosophila heat shock factor to arrays of a conserved $5 \mathrm{bp}$ unit. Cell 64: 585-593.

Yarus, M. 1992. Proofreading, NTPases and translation: Constraints on accurate biochemistry. Trends Biochem. Sci. 17: 130-133.

Zawel, L. and D. Reinberg. 1992. Advances in RNA polymerase II transcription. Curr. Opin. Cell Biol. 4: 488-495.

Zhu, H., A.L. Roy, R.G. Roeder, and R. Prywes. 1991. Serum response factor affects preinitiation complex formation by TFIID in vitro. New Biol. 3: 455-464. 


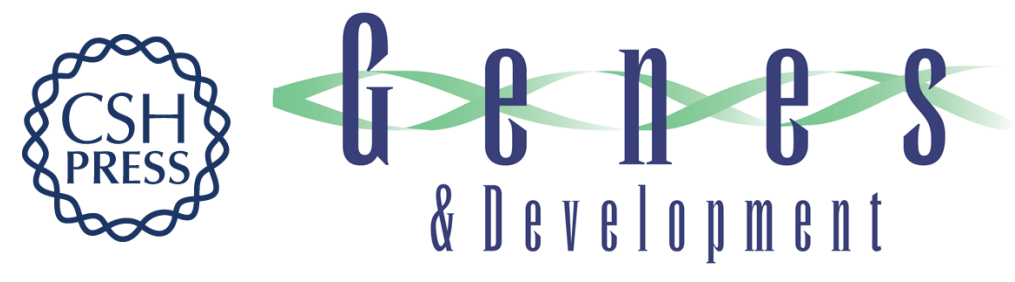

\section{Synergism in transcriptional activation: a kinetic view.}

D Herschlag and F B Johnson

Genes Dev. 1993, 7:

Access the most recent version at doi:10.1101/gad.7.2.173

References This article cites 51 articles, 21 of which can be accessed free at: http://genesdev.cshlp.org/content/7/2/173.full.html\#ref-list-1

License

Email Alerting Receive free email alerts when new articles cite this article - sign up in the box at the Service top right corner of the article or click here.

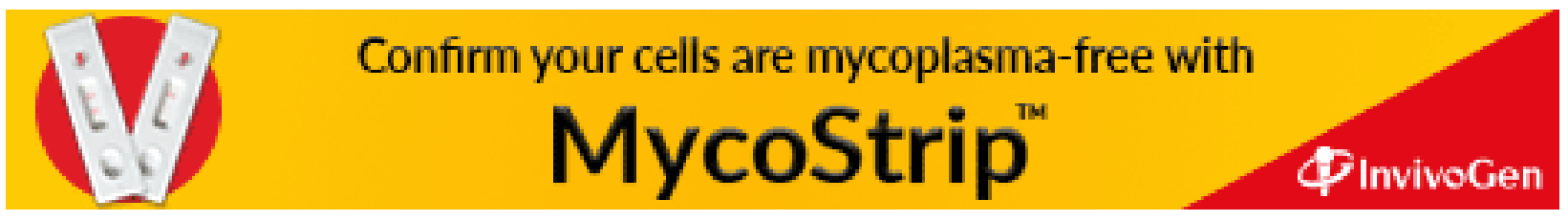

